\title{
Toughening of Syndiotactic Polystyrene and Poly(2,6-dimethyl-1,4-diphenylene oxide) Blends I. Influence of Mixing Protocol and Blend Conditions
}

\author{
Soo Hyun ChOI, Iwhan CHO, and Kwang Ung $\mathrm{KIM}^{*, \dagger}$ \\ Department of Advanced Materials Engineering (Polymerics Program), \\ Korea Advanced Institute of Science and Technology, \\ 207-43, Cheongrhangri-dong, Dongdaemoon-gu, Seoul 130-012, Korea \\ * Polymer Hybrid Research Center, Korea Institute of Science and Technology, \\ 39-1, Hawolgok-dong, Sungbuk-gu, Seoul 136-791, Korea
}

(Received January 23, 1999).

\begin{abstract}
It is well known that brittle polymers can be considerably toughened by the incorporation of miscible amorphous polymers or a thermoplastic elastomer. The effect of rubber toughening was studied via reactive extrusion to investigate the fracture mechanism and morphology controls of syndiotactic polystyrene (SPS)/poly(2,6-dimethyl-1,4diphenylene oxide) (PPO) blends. Successful rubber toughening was achieved in compatibilization using reactive extrusion. The reactive polystyrene (RPS) as a compatibilizer and styrene-ethylene/butylene-styrene block copolymer (SEBS) as an impact modifier were used in an extrusion experiment. The impact strength was highly dependent on the blending condition and the composition. The best result was obtained by 2-step mixing; the first mixing of PPO with RPS (1.0 phr oxazoline content) and functionalized SEBS (f-SEBS, $0.4 \mathrm{phr}$ maleic anhydride content) then followed with SPS mixing. Increase in RPS level gave rubber particle size reduction due to the reactions at the interfaces among polymer phases, as demonstrated by the decreased $\tan \delta$ value. The storage modulus $\left(E^{\prime}\right)$ of SPS/PPO blends was decreased with increasing PPO content.
\end{abstract}

KEY WORDS Syndiotactic Polystyrene/Poly(2,6-dimethyl-1,4-diphenylene oxide)/ Reactive Extrusion

/ Reactive Polystyrene / Rubber Toughening /

Numerous papers for toughening polymer blend systems have been reported using maleic anhydride (MAH) as a reactive modifier with various copolymers. ${ }^{1-4}$ Campbell et al. ${ }^{5}$ reported ductility improvements of polyamide 66 (PA66)/Poly(2,6-dimethyl-1,4diphenylene oxide) (PPO) blends with a styrene-ethylene/ butylene-styrene (SEBS) impact modifier by addition of an anhydride functionalized PPO copolymer, resulting in an enormous reduction of the dispersed particle size. Gallucci ${ }^{6}$ also studied polyamide (PA)/PPO blends compatibilized by an MAH grafted SEBS and reported improvement in tougheness of the blends.

Lazzeri and Bucknall ${ }^{7}$ reported that when the dispersed rubber particles were less than $0.13 \mu \mathrm{m}$, there was no cavitation in this toughening experiment with nylon/rubber system. They also concluded that no cavitation or even with some cavitation at very high stress would give very inefficient toughening effect. In the meantime, $\mathrm{Wu}^{8}$ explained in his interparticle theory that percolation overlapped by induced rubber particle stress could result in a rapid increase of impact strength. $\mathrm{He}$ also reported that easy deformation and sufficient toughening could be expected below the critical interparticle distance yielding plane-stress state of the matrix.

Since syndiotactic polystyrene (SPS) can be polymerized using the titanium and organoaluminum compounds by Ishihara et al., ${ }^{9}$ many researchers ${ }^{9-13}$ have studied polymerization conditions, polymorphism of SPS crystal, and blends with other polymers. Studies on SPS/PPO and SPS/ poly(vinyl methyl ether) (PVME) blends have focused on the crystallization behavior and miscibility of the blends. For instance, Guerra et al. ${ }^{10,12}$ have reported that the polymorphic behavior of melt

† To whom correspondence should be addressed. crystallized SPS can be changed by blending with PPO which strongly favors $\beta$-form. In the case of SPS/PVME blends, the influence of PVME on some morphological and kinetic properties of SPS have been reported by Cimmino et al. ${ }^{14}$ The system polystyrene (PS)/PPO has been known as a miscible pair. ${ }^{15}$ The SPS/PPO blend is also expected to be a miscible system, a model system in this study.

In order to improve the mechanical properties of immiscible polymer blends, compatibilizers such as block and graft copolymers have been widely used. It is because block and graft copolymers are very effective in reducing the interfacial tension and improve interfacial adhesion by chain entanglement or bridging at the interface. ${ }^{16-18}$

A grafted copolymer is formed by reaction of $\mathrm{MAH}$ to the rubber yielding ethylene-propylene rubber(EPR)graft-MAH or SEBS-graft-MAH. The amine end groups on the polyamide chain can also take MAH to form a grafted copolymer. These grafted copolymers can be used as compatibilizer in blends, which improves interfacial strength between polyamide phase and the dispersed rubber particles. ${ }^{19,20}$ We have studied the influence of composition, morphology, phase structure, and rheology of SPS/PPO blends with thermoplastic elastomers. The compatible SPS/PPO blends have been further toughened by incorporation of MAH functionalized SEBS and oxazoline functionalized PS.

Studies on SPS toughening and its blends with PPO are still in early stage, which is essential to understand the properties and applications of semicrystalline polymer SPS. There is no result reported on the toughening of SPS/PPO blend system yet. The toughened SPS/PPO blend will attract great interest to both industry and academia. Especially, the successful toughening of SPS/ PPO will open many commercial application areas by its inherent high temperature resistance. Fairly easy 
processability can be another advantage of the system.

\section{EXPERIMENTAL}

Synthesis of SPS and Characterization

The catalyst cyclopentadienyl titanium trichloride $\left(\mathrm{CpTiCl}_{3}\right)$ was commercial products (Aldrich Chem.). The cocatalyst was methylaluminoxane (MAO) from Akzo Nobel Ltd. The solvent toluene of extra pure grade (Junsei Co.) was further purified by distillation over sodium in vacuum. Styrene monomer was synthesis grade (Junsei Co.) and was purified by distillation in vacuum.

Polymerization was carried out in toluene as solvent. The catalyst $\mathrm{CpTiCl}_{3}$ was used at $60^{\circ} \mathrm{C}$ for $60 \mathrm{~min}$ and the $\mathrm{Al} / \mathrm{Ti}$ mole ratio was 2500 . An autoclave under vacuum was employed for polymerization and reaction was terminated by addition of $\mathrm{CH}_{3} \mathrm{OH} / \mathrm{HCl}$ solution then precipitated with excess $\mathrm{CH}_{3} \mathrm{OH}$. The product was dried at $80^{\circ} \mathrm{C}$ for $72 \mathrm{~h}$ under vacuum.

The fractionation was carried out by extracting the product boiling with 2-butanone in a Soxhlet-type apparatus to remove by-products such as isotactic polystyrene (IPS) and atactic polystyrene (APS).

$T_{\mathrm{g}}$ and $T_{\mathrm{m}}$ for the synthesized SPS were determined by a differential scanning calorimeter (DSC-7, PerkinElmer), $T_{\mathrm{g}}$ as the reflection point and $T_{\mathrm{m}}$ as the peak temperature of melting curve as shown in Table I.

Synthesized SPS had $233000 \bar{M}_{w}$ and 2.2 polydispersity index (PDI) as shown in Table I and Figure 1. The molecular weights and polydispersity were obtained by means of high temperature gel permeation chromatography (Waters Co.) in $o$-dichlorobenzene (Figure 1a). The columns were calibrated with a series of narrow distribution polystyrene standards. Also, ${ }^{13} \mathrm{C}$ NMR spectra of SPS were recorded at $110^{\circ} \mathrm{C}$ in 1,1,2,2-tetra chloroethane- $d_{2}$ using a spectrometer (Bruker-ARX300 ) at $135^{\circ} \mathrm{C}$ (Figure $1 \mathrm{~b}$ ).

\section{Materials and Blending}

Synthesized SPS from autoclave was obtained in powder form. PPO powder was received as a generalpurpose grade (646-111, General Electric Co.). Both powders were dried in a vacuum oven at $50^{\circ} \mathrm{C}$ for $24 \mathrm{~h}$ before blending.

The compatibilizer reactive polystyrene (RPS1005, Japan Catalyst Co.) contained $5 \mathrm{wt} \%$ oxazoline functional group. The impact modifier SEBS was a tri-block copolymer (Kraton G1652, Shell Chem. Co.) with ratio of styrene to ethylene/butylene (EB) block 29/71 by weight and the glass transition temperature of EB block $-42^{\circ} \mathrm{C}$. The $2 \mathrm{wt} \% \mathrm{MAH}$ grafted SEBS (f-SEBS) was

Table I. Properties of synthesized syndiotactic polystyrene

\begin{tabular}{cc}
\hline Property & Value \\
\hline$T_{\mathrm{g}}^{\mathrm{a}}$ & $102.1^{\circ} \mathrm{C}$ \\
$T_{\mathrm{m}}^{\mathrm{a}}$ & $271.0^{\circ} \mathrm{C}$ \\
$\bar{M}_{w}{ }^{\mathrm{b}}$ & $233000 \mathrm{~g} \mathrm{~mol}^{-1}$ \\
PDI & 2.2
\end{tabular}

${ }^{a} \mathrm{DSC}$ at a scan rate of $10^{\circ} \mathrm{C} \mathrm{min}^{-1}$. ${ }^{\mathrm{b}} \mathrm{GPC}$ at $135^{\circ} \mathrm{C}$.



(a)

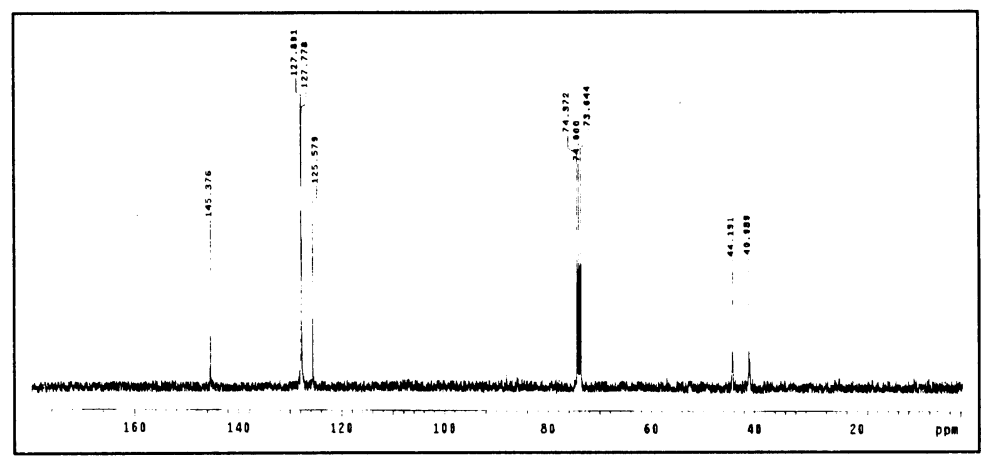

(b)

Figure 1. Gel permeation chromatography of synthesized SPS in $o$-dichlorobenzene at $135^{\circ} \mathrm{C}$ (a), and ${ }^{13} \mathrm{C}$ NMR spectrum in $1,1,2,2$ tetrachoroethane- $d_{2}$ at $135^{\circ} \mathrm{C}$ (b). 
Table II. Blend compositions, glass transition temperature, and impact strength

\begin{tabular}{|c|c|c|c|c|c|c|c|}
\hline \multirow{3}{*}{ Sample } & \multicolumn{4}{|c|}{ Composition } & \multirow{3}{*}{$\frac{T_{\mathrm{g}}}{{ }^{\circ} \mathrm{C}, \mathrm{DMA}}$} & \multirow{3}{*}{$\begin{array}{c}\begin{array}{c}\text { Impact } \\
\text { strength }\end{array} \\
10^{3} \mathrm{~J} \mathrm{~m}^{-1}\end{array}$} & \multirow{3}{*}{ Remark } \\
\hline & \multirow{2}{*}{$\begin{array}{c}\text { SPS } \\
w t \%\end{array}$} & \multirow{2}{*}{$\begin{array}{c}\mathrm{PPO} \\
w \mathrm{t} \%\end{array}$} & \multirow{2}{*}{$\begin{array}{c}\text { SEBS/f-SEBS }{ }^{\mathrm{a}} \\
\mathrm{phr}\end{array}$} & \multirow{2}{*}{$\frac{\text { RPS }^{\mathrm{a}}}{\mathrm{phr}}$} & & & \\
\hline & & & & & & & \\
\hline A & 100 & 0 & $0 / 0$ & 0 & 101 & 3.2 & \\
\hline B & 0 & 100 & $0 / 0$ & 0 & 204 & 7.2 & \\
\hline $\mathrm{C}$ & 70 & 30 & $0 / 0$ & 0 & 131.9 & 5.1 & \multirow{4}{*}{$\begin{array}{l}\text { 1-Step } \\
\text { mixing }\end{array}$} \\
\hline $\mathrm{D}$ & 70 & 30 & $0 / 20$ & 0 & 134.02 & 8.0 & \\
\hline $\mathrm{E}$ & 70 & 30 & $0 / 0$ & 5 & 139.4 & 5.1 & \\
\hline $\mathrm{F}$ & 70 & 30 & $0 / 20$ & 5 & 126.3 & 14.0 & \\
\hline G & 70 & 30 & $0 / 20$ & 0 & 133.16 & 8.5 & \multirow{8}{*}{$\begin{array}{l}\text { 2-Step } \\
\text { mixing }\end{array}$} \\
\hline $\mathrm{H}$ & 70 & 30 & $0 / 20$ & 5 & 118.3 & 23.5 & \\
\hline I & 70 & 30 & $0 / 20$ & 10 & 132.04 & 25.5 & \\
\hline $\mathrm{J}$ & 70 & 30 & $0 / 20$ & 15 & - & 26.5 & \\
\hline $\mathrm{K}$ & 70 & 30 & $0 / 20$ & 20 & 135.06 & 29.0 & \\
\hline $\mathrm{L}$ & 70 & 30 & $0 / 20$ & 25 & 123.58 & 26.9 & \\
\hline $\mathrm{M}$ & 70 & 30 & $20 / 0$ & 25 & 122.18 & 21.0 & \\
\hline $\mathrm{N}$ & 70 & 30 & $10 / 10$ & 20 & 127.04 & 16.0 & \\
\hline
\end{tabular}

${ }^{a}$ Part per hundred resin.

Kraton FG1901X from Shell. The blending was done with an intermeshing corotating twin-screw extruder (DSR 28-1, Prism) at $290^{\circ} \mathrm{C}, 50 \mathrm{rpm}$ screw a speed with fixed feed rate of $2.4 \mathrm{kgh}^{-1}$. Two blending methods were employed: 1-step and 2-step mixing. The 1-step mixing was that all ingredients were dry-blended first and then extruded. The 2-step mixing was that all ingredients except SPS were dry-blended and extruded. The pre-blended samples were then mixed with SPS by extrusion. All the samples used in the experiment were dried at $80^{\circ} \mathrm{C}, 6 \mathrm{~h}$ in vacuum. The blend compositions are listed in Table II.

\section{Mechanical Properties}

Izod impact tester (ITR 2000, Radmana) was used to measure impact strength. Impact specimens were molded as $76 \times 13 \times 3 \mathrm{~mm}^{3}$ using Mini-Max molder (CSI183MMV, Custom Scientific Instruments Inc.) with $50 \mathrm{rpm}$ for $5 \mathrm{~min}$ at $300^{\circ} \mathrm{C}$. Specimens have a $2.5 \mathrm{~mm}$ notch at the center. Impact test was carried out at room temperature.

Dynamic-mechanical thermal analyzer (DMA, Rheometrics MK III) was used to measure $T_{\mathrm{g}}$ and storage modulus $E^{\prime}$ of blends. Data were collected for bending mode at a frequency of $1 \mathrm{~Hz}$ and heating rate $4^{\circ} \mathrm{C}$ $\min ^{-1}$.

\section{Morphology}

A scanning electron microscopy (SEM, S-2500 and S-510, Hitachi) was used to observe the blend morphology. The molded specimens were freeze-fractured after quenching for $10 \mathrm{~min}$ in liquid nitrogen then taken SEM microphotograph. All fractured surfaces of the samples were furnished with gold coating.

\section{Rheology}

The rheological measurements were carried on a Rheometrics Dynamic Spectrometer (RDS 7700, Rheometrics Co.) on which a $12.5 \mathrm{~mm}$ diameter parallel plate with $1.0 \mathrm{~mm}$ gap size was employed. Frequency range was $0.1-500 \mathrm{rad} \mathrm{s}^{-1}$, strain $10 \%$, and $290^{\circ} \mathrm{C}$ temperature. Samples were dried overnight in a vacuum oven at $120^{\circ} \mathrm{C}$ under the nitrogen atmosphere before measurement.

\section{RESULTS AND DISCUSSION}

\section{Impact Strength}

Table II shows the glass transition temperature $T_{\mathrm{g}}$ and impact strength of SPS/PPO blends with various blending conditions. Dynamic mechanical analysis (DMA) data showed all SPS/PPO samples were indeed compatible blends exhibiting single $T_{\mathrm{g}}$. The differential scanning calorimetry (DSC) also confirmed this. The impact strength of compatible SPS/PPO blends had the value between those of pure materials, but no synergistic effect. To toughen the SPS/PPO blend further a tri-block copolymer SEBS and a reactive polystyrene (RPS) were tested with two different blending procedures. The 2-step mixing showed a big improvement in impact strength over the 1-step mixing. Increase in impact strength was even more dramatic with the reactive 


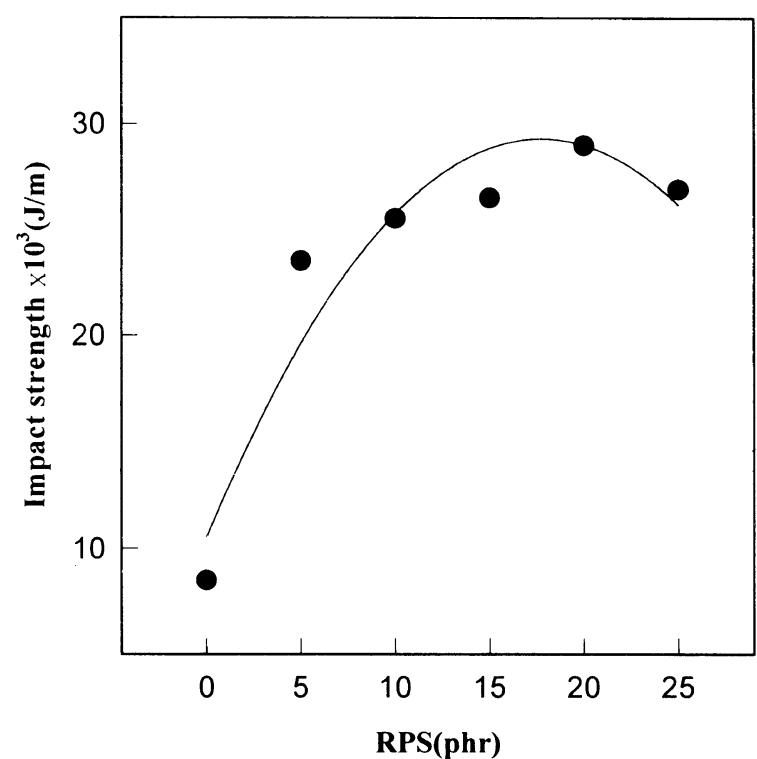

Figure 2. Impact strength of $\mathrm{SPS} / \mathrm{PPO} / \mathrm{f}-\mathrm{SEBS}=70 / 30 / 20 \quad(\mathrm{phr})$ blends varying RPS.

additives, f-SEBS and RPS. For example, at a fixed SPS/PPO ratio (70/30 by weight), the reactive f-SEBS at $20 \mathrm{phr}$ level gave some improvement (samples $\mathrm{C}$ and $\mathrm{D}$ in Table II) while RPS at 5 phr level did not improve the impact strength (samples $\mathrm{C}$ and $\mathrm{E}$ ) in 1-step mixing. However, when both f-SEBS (20 phr) and RPS (5 phr) were added simultaneously, the impact strength was increased to almost 3 times (sample F). This demonstrates that there are some synergistic effects of two reactive additives, even in 1-step mixing.

The 2-step mixing increased the impact strength of blends further, probably allowing the functional group to react with PPO first before SPS addition. In other words, MAH (f-SEBS) and oxazoline react with PPO improving interfacial adhesion between PPO and other added ingredients. Inherently compatible SPS/PPO blends would not be interfered by the interfacial reaction of functional group. In 2-step mixing, f-SEBS(20 phr) alone gave a marginal improvement in impact strength (samples D and G, Table II). However, presence of RPS $(5 \mathrm{phr})$ in SPS $/ \mathrm{PPO} / \mathrm{f}-\mathrm{SEBS}=70 / 30 / 20(\mathrm{phr})$ made a big increase in impact strength (samples $\mathrm{F}$ and $\mathrm{H}$ ), another evidence of synergy by two reactive additives. The non-reactive SEBS was inferior to the reactive f-SEBS (samples $\mathrm{K}$ and $\mathrm{M}$ ).

With SPS/PPO/f-SEBS $=70 / 30 / 20(\mathrm{phr})$ blends, effect of RPS on impact strength is shown in Figure 2. A big increase with $5 \mathrm{phr}$ can be seen but after $5 \mathrm{phr}$ impact strength is somewhat gradual and a maximum can be seen at $20 \mathrm{phr}$ level ( $1.0 \mathrm{phr}$ oxazoline level).

At fixed $20 \mathrm{phr}$ level, effect of SEBS/f-SEBS ratio in SPS $/ \mathrm{PPO} / \mathrm{RPS}=70 / 30 / 20(\mathrm{phr})$ is seen in Figure 3. As MAH content varies, a minimum impact strength is shown between $0.1-0.2 \mathrm{phr}$ and the highest impact is observed with $0.3-0.4 \mathrm{phr}$.

For the SPS $/ \mathrm{PPO}=70 / 30$ blend, the best impact strength could be achieved with $20 \mathrm{phr}$ f-SEBS $(0.4 \mathrm{phr}$ $\mathrm{MAH}$ ) and $20 \mathrm{phr}$ RPS (1.0 phr oxazoline), which gave almost 6-fold increase over the blend without two synergistic reactive additives.

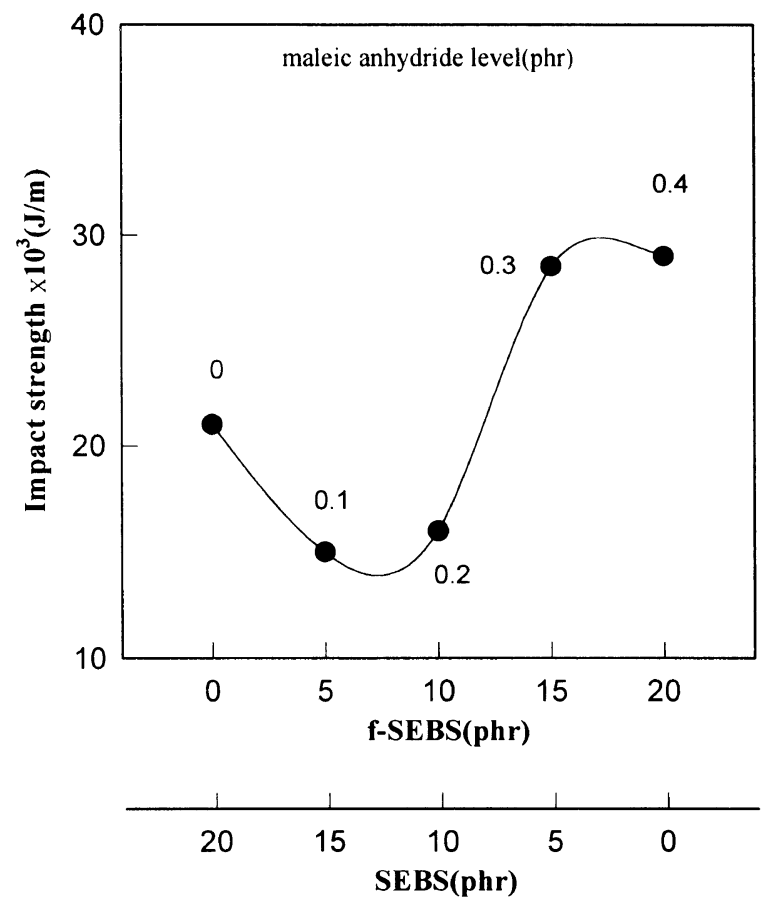

Figure 3. Impact strength of SPS/PPO/RPS (70/30/20 (phr)) varying SEBS/f-SEBS ratio at $20 \mathrm{phr}$ level.

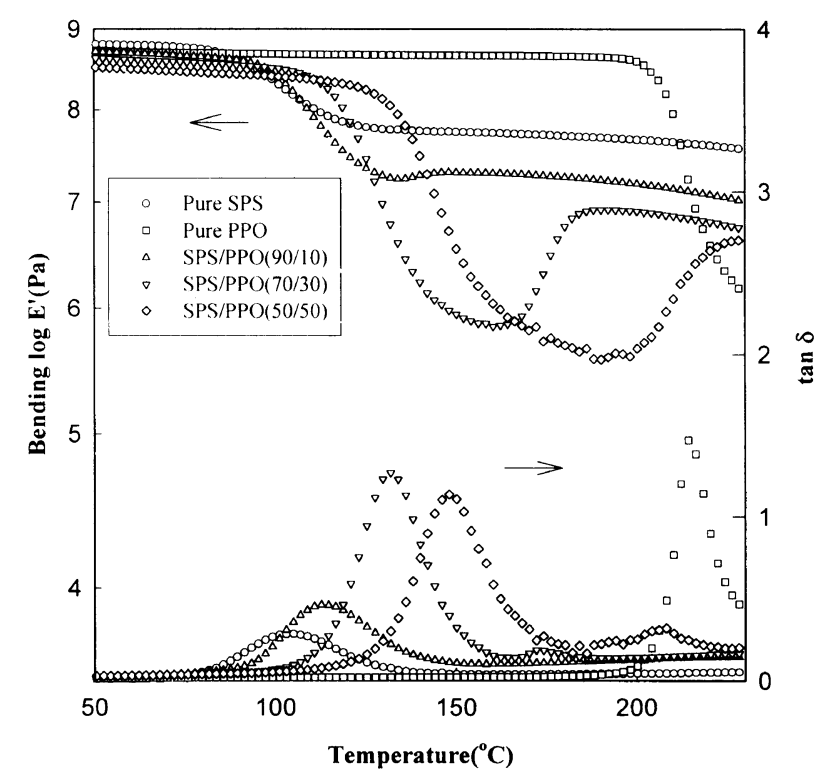

Figure 4. Storage modulus $E^{\prime}$ and loss tangent $\tan \delta$ for various SPS/PPO blends.

\section{Dynamic Mechanical Analysis (DMA)}

Figures 4 and 5 exhibit DMA data for various SPS/ PPO blends. Figure 4 shows that composition ratio in compatible SPS/PPO blend has a pronounced influence to storage modulus $E^{\prime}$ and $\tan \delta$. One can see that these blends are compatible and SPS $/ \mathrm{PPO}=70 / 30$ blend gives a big reduction in $E^{\prime}$ and a maximum $\tan \delta$ peak, implying that the blend has the most favorable morphology yielding the highest impact strength. Effects of mixing method and additives are shown in Figure 5. At fixed SPS $/ \mathrm{PPO}=70 / 30$ composition, RPS $(5 \mathrm{phr})$ or f-SEBS $(20 \mathrm{phr})$ alone hardly changed $E^{\prime}$ and $\tan \delta$. In fact, they made a slight shift to higher temperature. However, simultaneous presence of RPS and f-SEBS 
made $E^{\prime}$ reduced and $\tan \delta$ shifted to lower temperature. The 2-step mixing process gave the most reduction of $E^{\prime}$ and lowest $\tan \delta$ peak temperature. This $E^{\prime}$ reduction and $\tan \delta$ shift to lower temperature may imply the enhanced interfacial activity of functional groups, MAH and oxazoline. Thus MAH and oxazoline can be used to toughen SPS/PPO blends further.

The reduction of $E^{\prime}$ and $\tan \delta$ peak in the 2-step mixing over 1-step could be attributable to dispersion status of $\mathrm{f}$-SEBS in SPS/PPO continuous phase. In other words, the 2-step gave finer dispersion of rubbery material increasing interfacial area between matrix and dispersed phase, which contributed to lowering $E^{\prime}$ and $\tan \delta$. Increase of interfacial area in the 2-step mixing could be achieved easily by two extrusion passes giving smaller particle sizes of the dispersed phase. For example, in

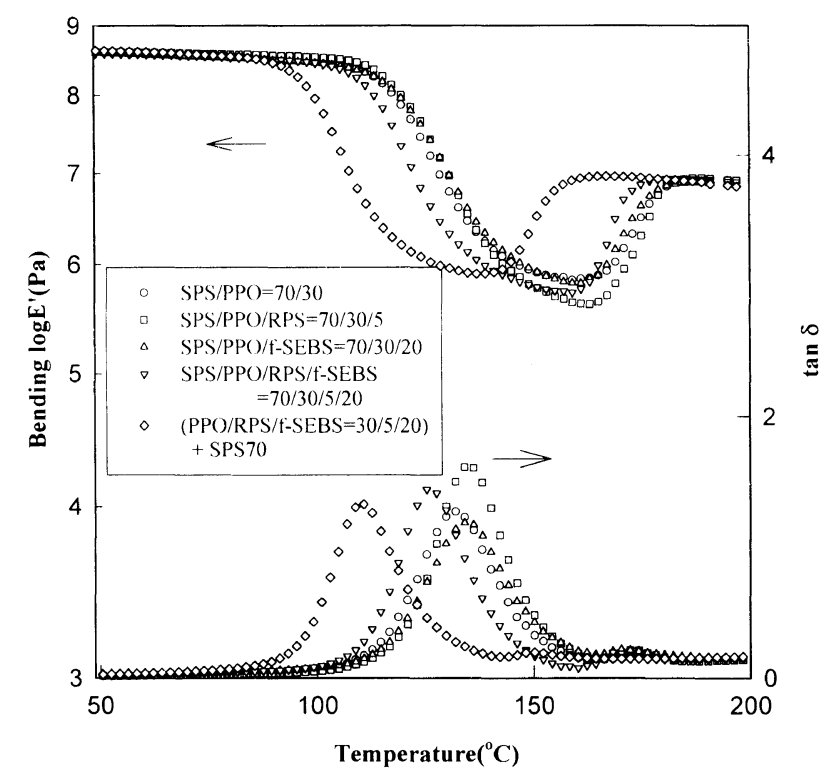

Figure 5. Storage modulus $E^{\prime}$ and loss tangent $\tan \delta$ for SPS $/ \mathrm{PPO}=$ $70 / 30$ blends varying blend conditions. same composition of SPS/PPO/RPS/f-SEBS $=70 / 30 / 5$ (phr)/20 (phr) system, the 2-step mixing lowered $E^{\prime}$ and $\tan \delta$ as shown in Figures 5 and 6.

\section{Morphology}

The 2-step mixing proved to be much superior over the 1-step mixing as demonstrated in Figure 6. In SPS/ $\mathrm{PPO} / \mathrm{f}-\mathrm{SEBS}=70 / 30 / 20(\mathrm{phr})$ system, the 1-step mixing produced rather fine and uniform particles $(\sim 0.25 \mu \mathrm{m})$ but with low impact strength of $8.0 \mathrm{~kJ} \mathrm{~m}^{-1}$ (Figure $6 \mathrm{a}$ ). It might be attributable to the fact of small particles with no creation of cavity as Lazzer and Bucknall ${ }^{7}$ suggested. When RPS ( 5 phr) was added to the above SPS/PPO/f-SEBS system, however, the dispersion became much coarse increasing particle size to $\sim 1.1 \mu \mathrm{m}$ (Figure 6b) but with improved impact strength to $14.0 \mathrm{~kJ} \mathrm{~m}^{-1}$. One could imagine that addition of reactive RPS would be located between immiscible SPS/PPO and f-SEBS phases increasing interfacial tension but interfering rubber dispersion, thus resulting in poor dispersion.

In the meantime, the 2-step gave finer and more uniform dispersion $(0.95 \mu \mathrm{m})$ than 1-step (Figures $6 \mathrm{~b}$ and $6 \mathrm{c})$. Further, the functionalized compatibilizers, RPS and f-SEBS, were well distributed between SPS and PPO phases. A careful inspection of microphotographs revealed that the added elastomeric additives, RPS and f-SEBS, were embedded in PPO phase, sometime together with SPS phase. This embedded morphology could be responsible for the enhanced impact strength even with coarser morphology (Figures $6 \mathrm{a}$ vs. $6 \mathrm{~b}$ and $6 \mathrm{c}$ ). Another reason could be that SEBS compatibilzer might be microphase-separated in PPO leading to ineffective impact modifier role.

Effect of RPS level is seen in Figure 7. With increasing RPS level, a finer dispersion can be achieved (Figures $7 \mathrm{a}$ and $7 \mathrm{~b}$ ). However, with the seemingly coarser dispersion of Figure $7 \mathrm{c}$, the highest impact strength $\left(29.0 \mathrm{~kJ} \mathrm{~m}^{-1}\right)$ can be attributable to the embedded morphology and

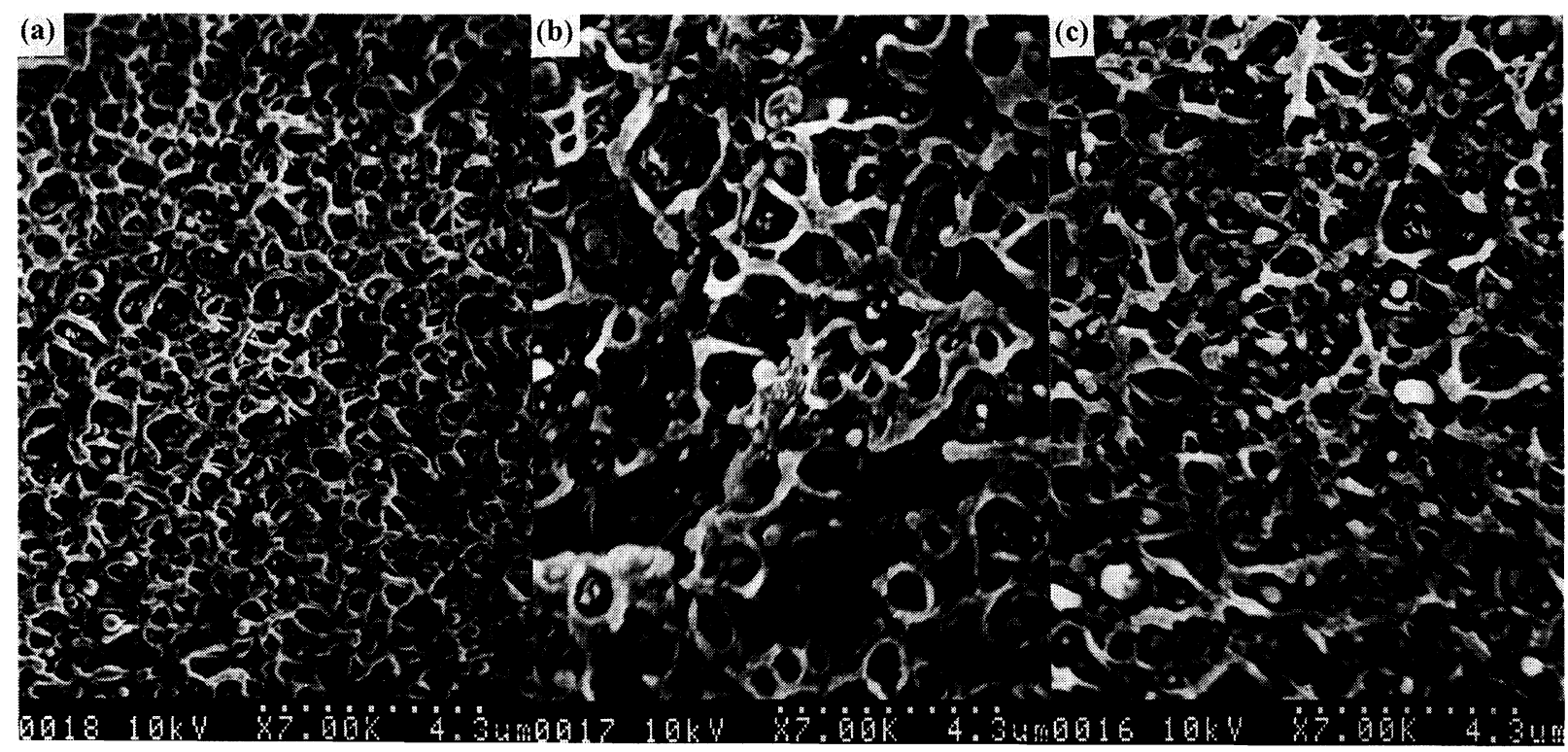

Figure 6. SEM microphotographs of fractured surface of SPS/PPO (70/30) blends at the blending condition. (a) SPS/PPO/f-SEBS (70/30/20 phr), (b) SPS/PPO/RPS/f-SEBS (70/30/5 phr/20 phr), and (c) PPO/f-SEBS/RPS (30/20 phr/5 phr) + SPS (70) Continuous phase (white portion) is SPS/PPO and the dispersed phase is f-SEBS. 


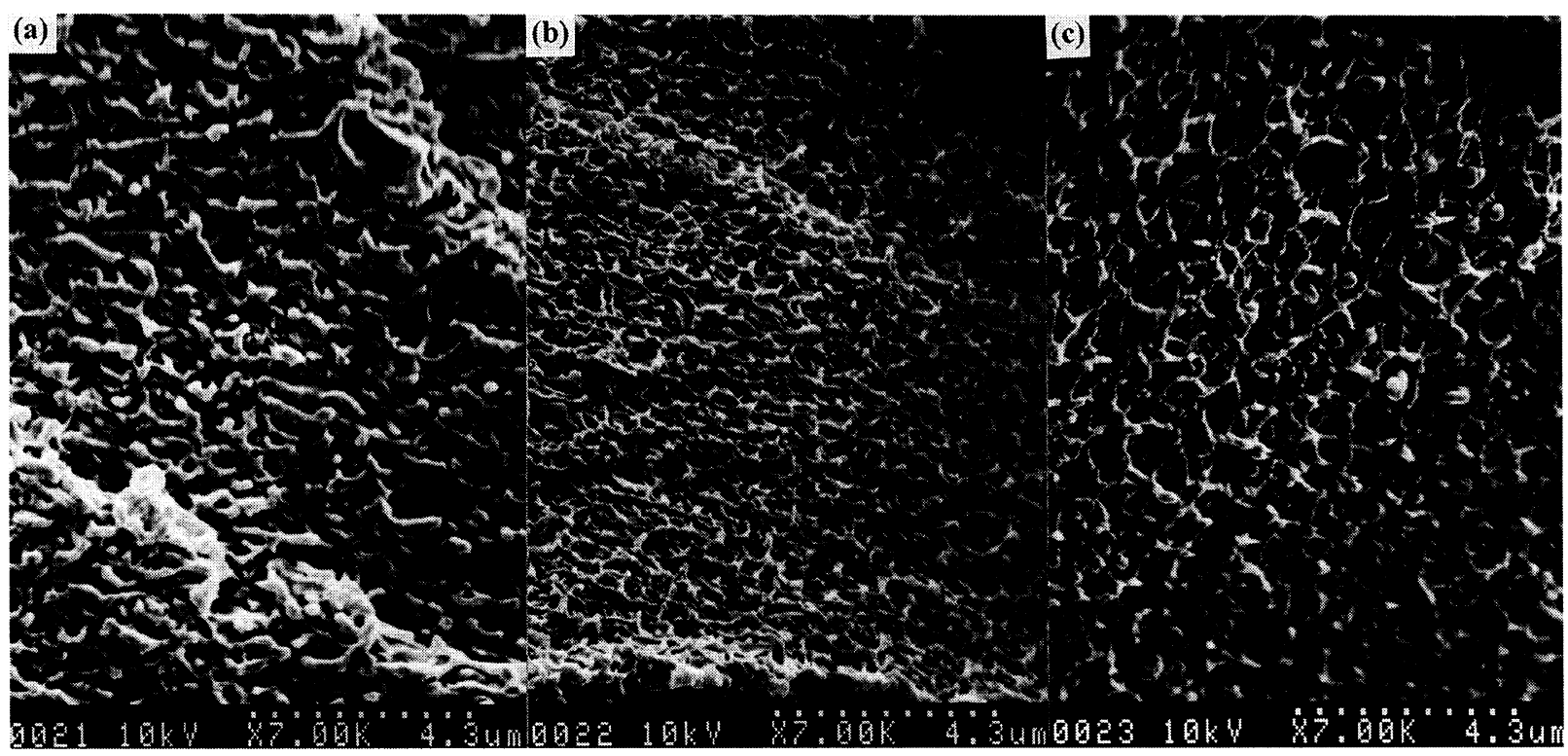

Figure 7. SEM microphotographs of fractured surface of SPS/PPO/f-SEBS (70/30/20 (phr) blends at the various RPS compositions in 2-step mixing (a) RPS $0 \mathrm{phr}$, (b) RPS $10 \mathrm{phr}(0.5 \mathrm{phr}$ oxazoline), and (c) RPS $20 \mathrm{phr}$ (1.0 phr oxazoline).

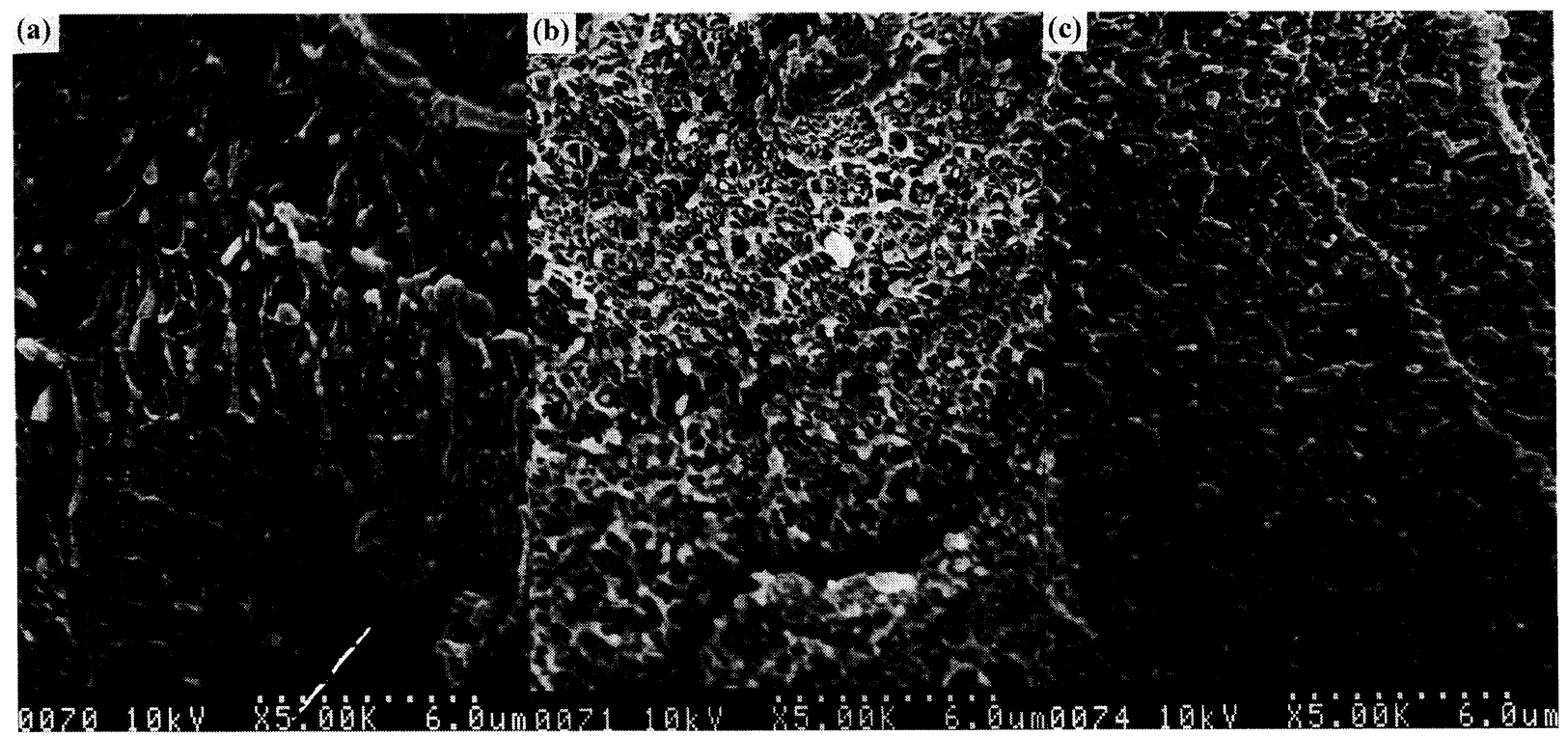

Figure 8. SEM microphotographs of fractured surface of SPS/PPO/RPS (70/30/20 phr) blends with 2-step mixing. (a) SEBS $20 \mathrm{phr}$ (MAH $0 \mathrm{phr}$ ), (b) SEBS $10 \mathrm{phr} / \mathrm{f}$-SEBS $10 \mathrm{phr}$ (MAH $0.2 \mathrm{phr}$ ), and (c) f-SEBS $20 \mathrm{phr}$ (MAH $0.4 \mathrm{phr}$ ). Continuous phase (white portion) is SPS/PPO and the dispersed phase is f-SEBS.

enhanced interfacial adhesion by oxazoline along with MAH in f-SEBS.

In the blend containing only as impact modifier with $\mathrm{SPS} / \mathrm{PPO} / \mathrm{RPS}=70 / 30 / 20 \mathrm{phr}$ (Figure 8a), impact strength was relatively high $\left(21.0 \mathrm{~kJ} \mathrm{~m}^{-1}\right)$ but morphology was rather irregular and coarse. It should be noted that Figure 8 a contained reactive compatibilizer RPS $20 \mathrm{phr}$ (1.0 phr oxazoline). During the mixing process, oxazoline group could be reacted with PPO yielding sufficient grafting to enhance impact strength. Addition of nonreactive SEBS would be microphase-separated in PPO during the first pass of extrusion. Subsequent second extrusion with SPS could not improve morphology. Examination of the morphology exhibited that SEBS was dispersed irregularly in SPS/PPO phase but the blend showed the tough fracture behavior resulting in enhanced impact strength as $\mathrm{Wu}^{8}$ explained. However, with SEBS(10 phr)/f-SEBS(10 phr) the morphology became much finer dispersion but impact strength was lowered somewhat $\left(16.0 \mathrm{~kJ} \mathrm{~m}^{-1}\right)$ (Figure $8 \mathrm{~b}$ ). Absence of non-reactive SEBS made the blend to be slightly bigger domain size but the highest impact strength could be achieved by good interfacial adhesion with two functional groups $\left(29.0 \mathrm{~kJ} \mathrm{~m}^{-1}\right)$ (Figure $8 \mathrm{c}$ ). Thus, good mechanical properties can be obtained by proper combination of finer morphology of dispersed rubber particles and good interfacial adhesion induced by functionalized compatibilizer.

\section{Rheology}

Figure 9 shows the dynamic storage modulus $G^{\prime}$ and complex viscosity $\eta^{*}$ for several SPS/PPO blends. Pure 


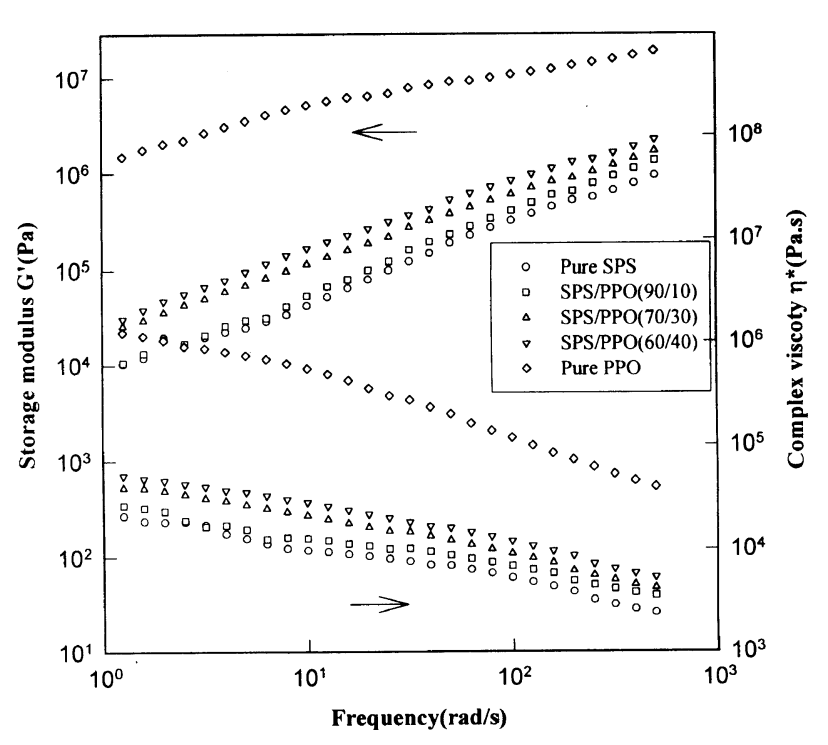

Figure 9. Dynamic storage modulus $G^{\prime}$ and complex viscosity $\eta^{*}$ for SPS, PPO, amd blends at $290^{\circ} \mathrm{C}$.

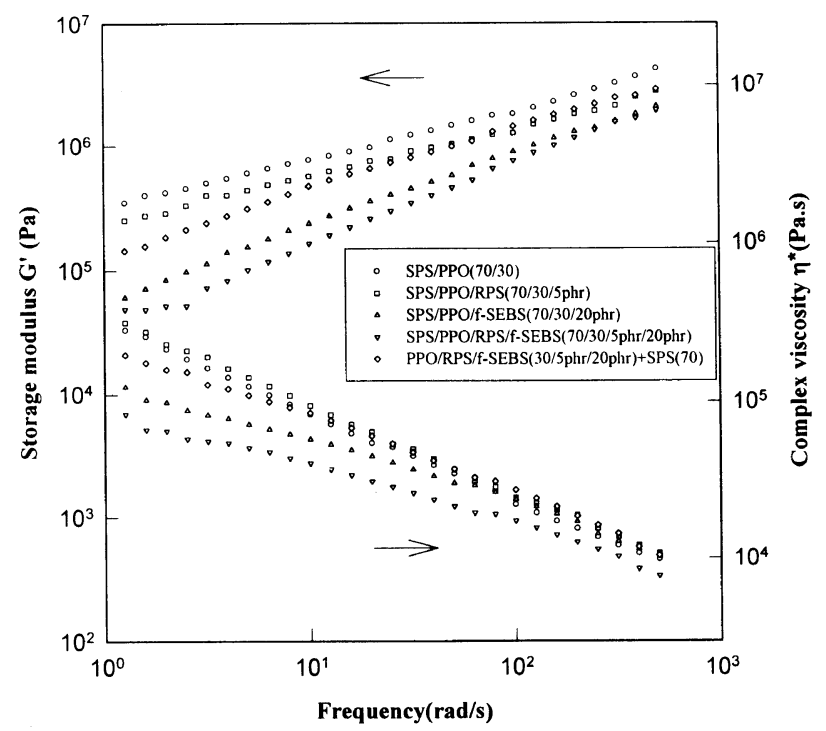

Figure 10. Dynamic storage modulus $G^{\prime}$ and complex viscosity $\eta^{*}$ for various SPS/PPO blends at $290^{\circ} \mathrm{C}$.

PPO has much higher $G^{\prime}$ and $\eta^{*}$ than pure SPS. Since SPS/PPO blends in Figure 9 were all SPS-rich systems with miscible SPS/PPO continuous phase, $G^{\prime}$ and $\eta^{*}$ were close to those of SPS. Thus the compatible SPS/ PPO blends would give easy processability utilizing higher mechanical properties and heat resistance of PPO than SPS.

Two reactive compatibilizers, RPS and f-SEBS, were found to be very effective to improve impact strength of SPS/PPO blends, almost 6-fold increase. At fixed $\mathrm{SPS} / \mathrm{PPO}=70 / 30$ blend, RPS $(5 \mathrm{phr})$ reduced $G^{\prime}$ slightly but increased $\eta^{*}$ slightly as shown in Figure 10. On the other hand, f-SEBS (20 phr) gave big reduction in $G^{\prime}$ and $\eta^{*}$. Even further reductions in $G^{\prime}$ and $\eta^{*}$ could be observed by adding both RPS and f-SEBS simultaneously in 1-step mixing. Low $G^{\prime}$ and $\eta^{*}$ may be due to effective compatibilization of additives during extrusion process. By allowing more time for functional groups to react with 2-step blending process (2-extrusion passes), $G^{\prime}$ and $\eta^{*}$ became very close to SPS/
$\mathrm{PPO}=70 / 30$ blend. $G^{\prime}$ for 2 -step mixing remains a little lower than SPS $/ \mathrm{PPO}=70 / 30$ blend but $\eta^{*}$ at lower frequency is a little higher than SPS $/ \mathrm{PPO}=70 / 30$ as seen in Figure 10. The higher $G^{\prime}$ and $\eta^{*}$ for the 2-step than the 1-step may imply that the functional groups, $\mathrm{MAH}$ and oxazoline, react at the interface between PPO and additives resulting in slightly higher molecular weight.

\section{CONCLUSIONS}

The compatible SPS/PPO blends can be further toughened by addition of functionalized additives. The non-reactive SEBS, MAH functionalized f-SEBS, and oxazoline functionalized RPS give marginal improvement in impact strength when added individually. However, each functionalized compatibilizer yields better result than the non-reactive SEBS.

Blending f-SEBS and RPS simultaneously with the 2-step extrusion process has resulted in dramatic enhancement of impact strength, almost 6-fold increase. In the first pass of reactive extrusion process, functional groups $\mathrm{MAH}$ and oxazoline play a role to increase molecular weight by reacting with PPO producing good interfacial adhesion. During the second pass of reactive extrusion with SPS, the unreacted portion of functional groups can proceed further reaction at the interface of PPO and SPS. Since SPS is major continuous phase, there is strong possibility of interfacial reaction by the functional group, further enhancing compatibility and mechanical property.

Dynamic mechanical analysis, observation of phase morphology by SEM, and measurement of rheology have confirmed the improved impact strength by two functionalized comaptibilizers. Thus, the successful toughening of inherently compatible SPS/PPO blends can create many application areas by virtue of excellent mechanical properties and high heat resistance. Further, relatively low viscosity of SPS/PPO blends would provide easy processability, enabling to produce a fairly complex shape easily.

\section{REFERENCES}

1. S. Y. Hobbs, M. E. J. Dekkers, and V. H. Watkins, J. Mater. Sci., 24, 1316 (1989); S. Y. Hobbs, M. E. J. Dekkers, and V. H. Watkins, J. Mater. Sci., 24, 2025 (1989).

2. Y. C. Lai, J. Appl. Polym. Sci., 54, 1289 (1994).

3. K. Suzuki and S. Ono, Japanese Patent, No. 60-155259 (1985).

4. M. Xanthos and S. S. Dagli, Polym. Eng. Sci., 31, 929 (1991); M. Xanthos, "Reactive Extrusion," Hanser Publishers, New York, N.Y., 1992.

5. J. R. Campbell, S. Y. Hobbs, T. J. Shea, and V. H. Watkins, Polym. Eng. Sci., 30, 1056 (1990).

6. R. R. Gallucci, US Patent, No. 5,260,37 (1993).

7. A. Lazzeri and C. B. Bucknall, J. Mater. Sci., 28, 6799 (1993).

8. S. Wu, Polymer, 26, 1855 (1985); A. Margolina and S. Wu, Polymer, 29, 2170 (1988).

9. N. Ishihara, T. Seimiya, M. Kuramoto, and M. Uoi, Macromolecules, 19, 2464 (1986); N. Ishihara, M. Kuramoto, and M. Uoi, EP 210615 (1986) ; N. Ishihara, M. Kuramoto, and M. Uoi, Macromolecules, 21, 3356 (1988).

10. G. Guerra, V. M. Vitagliano, C. De Rosa, P. Vittorio, Y. Chatani, Y. Shimane, T. Inagaki, T. Ijisu, T. Yukinari, and H. Shikuma, Polymer, 34, 1620 (1993).

11. F. de Candia, A. Ruvolo Filho, and V. Vittoria, Colloid Polym. Sci., 269, 650 (1991).

12. G. Guerra, C. De Rosa, and V. M. Vitagliano, Polym. Commun., 
32, 30 (1991).

13. V. Vittoria, F. de Candia, P. Iamelli, and A. Immirizi, Makromol. Chem., Rapid Commun., 9, 765 (1988).

14. S. Cimmino, E. Di Pace, E. Martuscelli, C. Silvestre, D. M. Rice, and F. E. Karasz, Polymer, 34, 214 (1993) ; S. Cimmino, E. D Pace, E. Martuscelli and C. Silvestre, Polymer, 34, 2799 (1993).

15. D. R. Paul and S. Newman Ed., "Polymer Blends," Academic Press, New York, N.Y., 1978, p 186; O. Olibisi, L. M. Robeson, and M. T. Shaw, "Polymer-Polymer Miscibility," Academic Press, New York, N.Y., 1979; J. Plans, W. J. MacKnight, and F. E. Karasz, Macromolucules, 17, 10 (1984).

16. P. S. Tucker, J. W. Balow, and D. R. Paul, Macromolecules, 21,
2794 (1988); J. C. Angola, Y. Fujita, T. Sakai, and T. Inoue, $J$. Polym. Sci., Polym. Phys. Ed., 26, 807 (1988); H. T. Patterson, K. H. Hu, and T. H. Grindstaff, J. Polym. Sci., 34, 31 (1991).

17. H. R. Brown, Macromolecules, 22, 2859 (1989).

18. C. Creton, E. J. Kramar, and G. Hadziioannou, Macromolecules, 24, 1846 (1991).

19. A. J. Oshinski, H. Keskkula, and D. R. Paul, Polymer, 33, 268 (1992); A. J. Oshinski, H. Keskkular, and D. R. Paul, Polymer, 37, 4919 (1996).

20. S. Y. Hobbs, R. C. Bopp, and V. H. Watkins, Polym. Eng. Sci., 23, 380 (1983). 